\title{
Parametric Modeling of Wave Propagation in Gas Mixtures - A System Identification Approach
}

\author{
Jesper Martinsson*, (student member) and Johan E. Carlson, (member) \\ EISLAB, Dept. of Computer Science and Electrical Engineering, Luleå University of Technology, SE-971 87 Luleå, Sweden. \\ *Email: Jesper.Martinsson@csee.ltu.se
}

\begin{abstract}
In ultrasonic pulse-echo systems, the observable properties are restricted to frequency dependent attenuation and phase velocity, which in turn are related to the material properties of the investigated media. In this paper we present a parametric model of the bulk modulus, composed of the basic physical building blocks of absorption (relaxation blocks). The parametric model is capable of handling the combined effect of the absorption mechanisms present in a gas or gas mixture and captures the experimental variation in the observation. The performance of the parametric model is demonstrated with pulse-echo experiments in oxygen, ethane and mixtures of the two. Compared to standard Fourier analysis techniques, the parametric model gives higher accuracy when estimating attenuation and phase velocity, and physical parameters such as relaxation strengths and relaxation frequencies can be extracted from it.
\end{abstract}

\section{INTRODUCTION}

Using ultrasonic techniques, the measurable properties of a gas are characterized by the complex frequency dependent wave number. The wave number is directly related to the bulk modulus, which for a non-dispersive and absorption free gas is a real-valued constant containing the equilibrium density and the thermodynamic speed of sound. For dispersive media (e.g. gases and gas mixtures), the relationship between the acoustic pressure and the condensation is dynamic, and the bulk modulus connecting these two quantities is both complexvalued and frequency dependent.

The standard approach to describe the dynamics of the bulk modulus is to parameterize it given the complete physical knowledge of the gas properties under investigation. However, for a gas or gas mixture with complex dynamic behavior and/or unknown components, a complete physical description of the complex bulk modulus is generally not available. For these situations a common approach is to use non-parametric methods, to describe the dynamics. Although non-parametric techniques are easy to apply, they give only moderately accurate descriptions. To obtain higher accuracy, parametric models must be used. When choosing the model structure, two things are desirable: First, the model should capture the dynamics of the system and second, the model should give information about the underlying physical properties.

In this paper, the bulk modulus is parameterized using a rational transfer function (spring-dashpot model). This structure has a well-known connection to some physical properties, while still keeping the number of parameters reasonably low. We use system identification techniques to estimate the parameters and cross-validation to prevent over-parametrization.
The model is validated by analyzing the prediction errors which show that the prediction errors are uncorrelated with the measured echoes. This means that the parametric model is able to capture the dynamics of the true system.

The proposed method is compared with standard nonparametric techniques using pulse-echo measurements in ethane, oxygen, and mixtures of the two. The experimental results show that the variances of the estimates are considerably smaller using the proposed method compared to nonparametric methods, especially for noisy data.

\section{THEORY}

In this section we start by reviewing the main absorption mechanisms present in a gas, and present them as equivalent spring-dashpot representations of the bulk modulus. A more general model structure is also presented, which is capable of handling the combined effect of all mechanisms. Next, the identification procedure is explained for pulse-echo experiments, followed by a description of optimization and initial values.

\section{A. Absorption}

In any real acoustic wave there are losses, acoustic energy is dissipated and converted into thermal energy. Sound absorption in a gas or gas mixture is described by several mechanisms. On a macroscopic level there is the classical absorption component, due to losses from viscosity and heat conduction. On a microscopic level, losses arise from relaxation of vibrationally and rotationally excited molecules. Each absorption mechanism contributes to a dissipation behavior with a corresponding relaxation time (or frequency). In gas mixtures, the behavior and relaxation time depend in a complex manner on the: molar fraction and molecular structure of each gas component in the mixture, temperature, pressure, and ultrasound frequency.

The absorption mechanisms are described by the frequency dependent bulk modulus, a material function that describes the dynamic relationship between the acoustic pressure and the condensation. In the frequency domain the relationship can be expressed as (assuming linearity and time invariance)

$$
P(\omega)=B(\omega) S(\omega),
$$

where $P(\omega)$ and $S(\omega)$ are the Fourier transforms of the acoustic pressure $p(t)$ and condensation $s(t)$, respectively. $B(\omega)$ is the frequency dependent bulk modulus. 


\section{B. Spring-Dashpot Representation}

In this paper, we represent the bulk modulus as a series of spring-dashpot sub-models connected i parallel to capture the variety of absorption mechanisms present in a real fluid.

In this representation, the acoustic pressure $p$ is considered the effort (force) variable, and the time derivative of the condensation $\dot{s}$ represents the flow (velocity) variable. Each sub-model contributes to a single relaxation peak at a given relaxation frequency, and is therefore an ideal building block to model the absorption mechanisms mentioned earlier. To motivate the use of this type of interconnecting sub-models, we review some of the absorption mechanisms present in a gas, in a spring-dashpot analogy.

For a lossless fluid, the bulk modulus is a real-valued constant composed of the equilibrium density and the thermodynamic speed of sound $B=\rho_{0} c_{0}^{2}$. The equivalent springdashpot representation is a simple spring with $k=\rho_{0} c_{0}^{2}=B$, see Fig. 1(a).

To model a fluid with viscous losses, a resistive element (dashpot) is added in parallel with the spring, see Fig. 1(b). For this spring-dashpot model the following dynamic relationship is obtained

$$
p(t)=k s(t)+\eta \dot{s}(t) .
$$

The equivalent frequency domain representation is obtained using Eq. (1) and $B(\omega)=B_{0}\left(1+\tau_{0} j \omega\right)$, where $B_{0}=k=$ $\rho_{0} c_{0}^{2}$ is the bulk modulus of the lossless wave, $\tau_{0}=\eta / k$ is the relaxation time, $\eta=4 / 3 \eta_{S}+\eta_{B}$ the dash-pot constant (viscosity), and $k$ the spring constant. The corresponding relaxation frequency is $\omega_{0}=\tau_{0}^{-1}$. Combining Eq. (2) with Euler's equation and the linear continuity equation, the result is the Navier-Stokes equation (excluding turbulence and vorticity), see [1].

A fluid that exhibits absorption due to a molecular relaxation mode, can be represented as the spring-dashpot model in Fig. 1(c). The dynamic relationship between $p$ and $s$ is

$$
p(t)\left(1+\frac{k_{2}}{k_{1}}\right)+\dot{p}(t) \frac{\eta}{k_{1}}=k_{2} s(t)+\eta \dot{s}(t)
$$

or using Eq. (1) and

$$
B(\omega)=B_{0} \frac{1+\tau_{s} j \omega}{1+\tau_{p} j \omega},
$$

where $B_{0}=k_{1} k_{2} /\left(k_{1}+k_{2}\right)=\rho_{0} c_{0}^{2}, \tau_{s}=\eta / k_{2}$ and $\tau_{p}=$ $\eta /\left(k_{1}+k_{2}\right)$. The corresponding relaxation frequency is $\omega_{0}=$ $\left(\tau_{s} \tau_{p}\right)^{-1 / 2}$. Notice that the spring-dashpot model in Fig. 1(c) is constructed by connecting the models in Fig. 1(b) and (a) in series, and can thus represent both.

In a gas or gas mixture several molecular relaxation mechanisms might be present together with absorption from the classical components. For this situation, the absorption have to be explained by a distribution of relaxation peaks. This behavior is modeled by considering a series of $M$ submodels connected in parallel, see Fig. 2, where each sub-model contributes to one relaxation peak. The bulk modulus for this

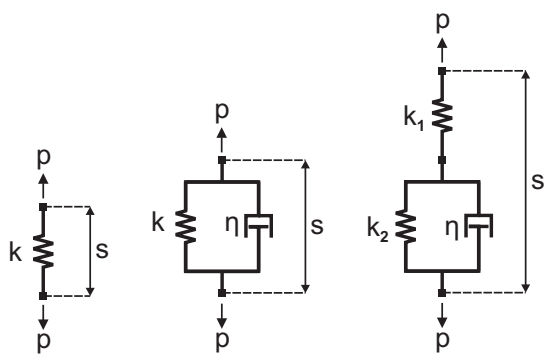

(a)

(b)

(c)

Fig. 1. Spring-dashpot representation of: (a) no absorption; (b) absorption due to viscosity; (c) absorption due to a molecular relaxation mode.

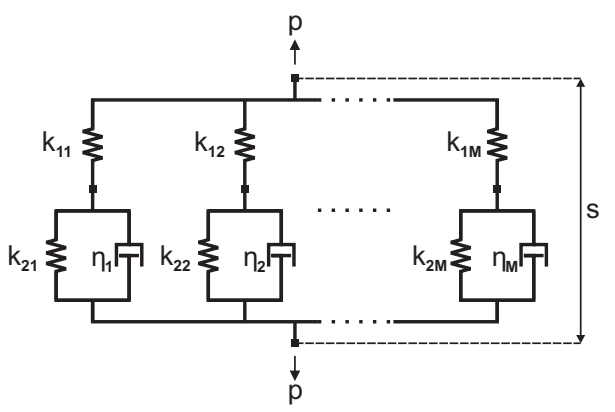

Fig. 2. Spring-dashpot model of the combined absorption mechanisms present in a gas or gas mixture. The model is constructed of $M$ sub-models connected in parallel.

type of model is

$$
B(\omega)=\frac{B_{0}}{M} \sum_{m=1}^{M} \frac{1+\tau_{s m} j \omega}{1+\tau_{p m} j \omega},
$$

where $\tau_{s m}=\eta_{m} / k_{2 m}$ and $\tau_{p m}=\eta_{m} /\left(k_{1 m}+k_{2 m}\right)$. The corresponding relaxation frequencies are $\omega_{0 m}=\left(\tau_{s m} \tau_{p m}\right)^{-1 / 2}$.

\section{Wave Propagation}

In one-dimensional wave propagation experiments, Eq. (1) is combined with Euler's equation and the linear continuity equation, and the following ordinary differential equation (ODE) is obtained,

$$
\frac{\partial^{2} P(x, \omega)}{\partial x^{2}}=-\frac{\rho_{0} \omega^{2}}{B(\omega)} P(x, \omega),
$$

where $x$ and $\rho_{0}$ are the position and equilibrium density. The ODE has the solution

$$
P(x, \omega)=A_{1}(\omega) e^{-j \beta(\omega) x}+A_{2}(\omega) e^{j \beta(\omega) x},
$$

where the complex wave number $\beta(\omega)=\sqrt{\rho_{0} \omega^{2} / B(\omega)}$. $A_{1}(\omega)$ and $A_{2}(\omega)$ are determined by the boundary conditions, i.e the amplitude of the wave at $x=0$. For a pulse-echo system, see Fig. 3, we can simplify the solution to one wave moving in one direction (assuming near field, perfect reflectivity and short pulses compared to the traveling distance). Knowing the acoustic pressure at two different locations, say at $x=0$ from the first echo and at $x=2 d$ from the second echo, 
where $d$ is the distance between the transducer and reflector, Eq. (7) can be written as

$$
P(2 d, \omega)=P(0, \omega) e^{-j \beta(\omega) 2 d},
$$

where $P(0, \omega)$ and $P(2 d, \omega)$ are the Fourier transforms of the first- and second echoes, respectively.

\section{System Identification}

In this paper the model in Eq. (5) (see Fig. 2) is used to describe the bulk modulus. The unknown model parameters are estimated in the discrete frequency domain, using Eq. (8) (pulse-echo experiments) and a least-squares (LS) fit given by

$$
J(\boldsymbol{\theta})=\sum_{l=-N / 2}^{N / 2-1}\left|P\left(2 d, \Omega_{l}\right)-P\left(0, \Omega_{l}\right) e^{-j \omega_{l}}{\sqrt{\frac{\rho_{0}}{B\left(\omega_{l}, \boldsymbol{\theta}\right)}}}^{2 d}\right|^{2},
$$

for an even $N$. Here $P\left(\cdot, \Omega_{l}\right)$ is the discrete Fourier transform (DFT) of a sampled echo, $\Omega_{l}=\omega_{l} / F_{s}$ is the discrete angular frequency and $F_{s}$ the sampling frequency. The parameter vector $\boldsymbol{\theta}=\left[B_{0}, \tau_{s 1}, \tau_{p 1}, \cdots, \tau_{s M}, \tau_{p M}\right]^{T}$ represent the unknown model parameters to be estimated by minimizing Eq. (9).

To estimate the appropriate number of sub-models $M$, cross-validation is used [2], [3]. Cross-validation evaluates the predictive performance when models of different sizes are confronted with new data sequences (other than those used for model estimation). Evaluating models with fresh data prevents over-parametrization and finds the appropriate model size within the chosen model structure.

To make sure that the model is capable of describing the true system, we have to validate its performance. The key concept here is to examine the prediction errors or "leftovers", i.e. the part of the ultrasonic pulses that the model could not predict, to determine the model's validity, see [3] .

\section{E. Optimization}

Minimizing Eq. (9) with respect to $\boldsymbol{\theta}$ results in a non-linear least-square (NLS) minimization problem, and good initial values are important. In this paper the initial values for the relaxation frequencies are obtained using the constant- $Q$ model [4], where the $(M)$ relaxation peaks are equally distributed along the $\log (\omega)$ axis covering the frequency range of interest.

In the identification step, the equilibrium density $\rho_{0}$ is treated as unknown and for simplicity it is incorporated with $B_{0}$ as a new parameter $\tilde{B}_{0}=B_{0} / \rho_{0}$, see Eq. (5) and Eq. (9). The initial value for $\tilde{B}_{0}$ is estimated with cross-correlation due to its relationship with the phase velocity at DC $(\omega=0)$. To minimize Eq. (9) the Nelder-Mead simplex (direct search) method is used [5].

\section{EXPERIMENTAL SETUP}

For all the experiments in this paper, the ultrasound echoes were measured in a pulse-echo configuration (see Fig. 3). An ultrasound transducer was mounted in a measurement cell, transmitting pulses through the gas towards a stainless steel reflector. The measurement cell was then immersed into the

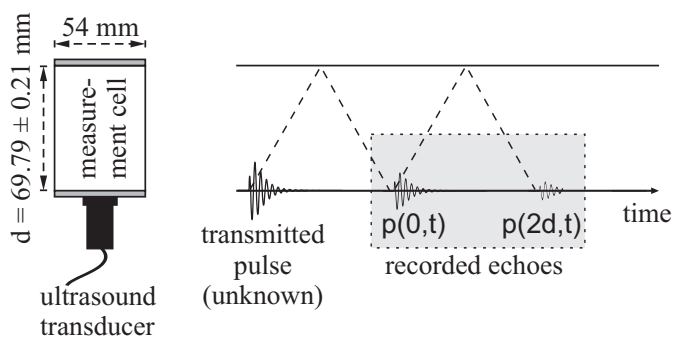

Fig. 3. The measurement cell and the pulse-echo principle. The transducer emits an unknown sound wave. The reflection from the bottom of the measurement cell is then recorded. Consecutive echoes can be recorded, as indicated in the figure.

pressure chamber. The whole setup was then placed in a temperature controlled chamber (Heraeus Vötsch HT4010), where keeping the temperature constant at the desired temperature $\left(20^{\circ} \mathrm{C}\right)$.

A custom-built pressure chamber was used to achieve a static pressure. Since the attenuation is extremely high at low pressures and high frequencies, we were limited to make measurements at high pressures (around 6.6 bar). The pressure in the chamber was measured with an ANDERSON TPP Pressure Transmitter.

A $1 \mathrm{MHz}$ air transducer with a effective diameter of $15 \mathrm{~mm}$ was used. For this transducer and the propagation distance, diffraction losses were assumed to be negligible [6]. To excite and receive acoustic pulses from the transducer, a Panametrics Pulser/Receiver Model 5072 was used.

All pulses were sampled at $100 \mathrm{MHz}$ with an 8-bit Tektronix $T D S 724,1 \mathrm{GHz}$ digitizing oscilloscope. For each measurement, the temperature was recorded using a PT100 sensor.

\section{RESUlTS}

In this section modeling and estimation results are presented, using the ultrasonic pulse-echo technique described in the previous section.

\section{A. Model Selection and Validation}

The model selection and validation results are presented for measurements in oxygen (highest SNR). Similar results are obtained for ethane and mixtures of the two.

The appropriate number of sub-models is determined by comparing the value of the criterion of fit in Eq. (9) using fresh data, for estimated models of different orders (crossvalidation). For models with $M \geq 4$ no significant decrease can be observed, see Fig. 4. For this reason a model order of $M=4$ is chosen.

Once the appropriate model size is determined and the parameters are estimated, the model is validated by analyzing its predictive performance. In Fig. 5 measured and predicted echoes are shown together with the residual (their difference). Note the scale differences $i$ the figure.

In the residual plot, a slight systematic variation can be noticed. However, testing its dependence with the first echo, by analyzing their cross-correlation, shows uncorrelation (independence) within a 99\% confidence region, see Fig. 6. 


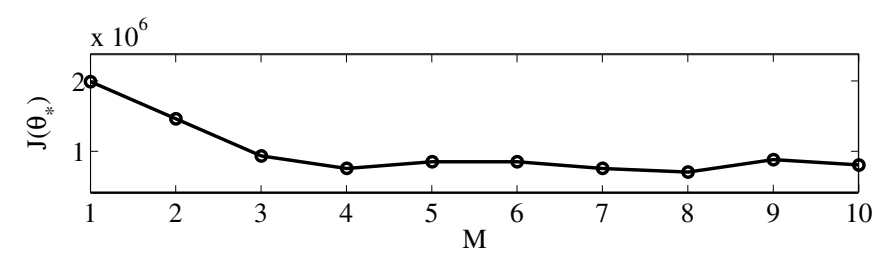

Fig. 4. Cost-function $J$ in Eq. (9) evaluated with fresh validation data, for estimated parameters $\boldsymbol{\theta}_{*}$ of different orders $M$.

(a)

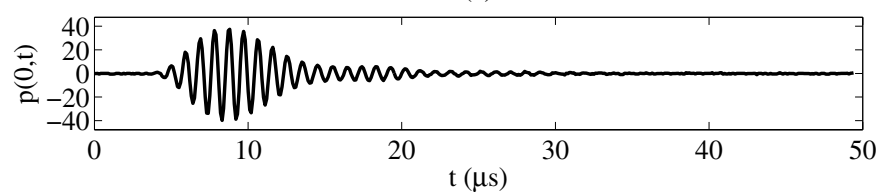

(b)

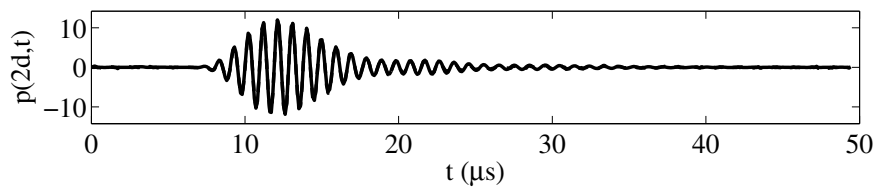

(c)

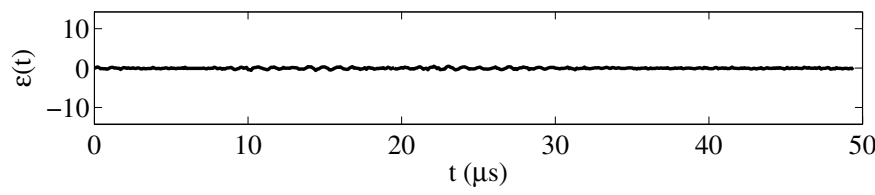

Fig. 5. Measured and predicted echoes with corresponding residual, using $M=4$ sub-models: (a) measured primary echo; (b) two (coinciding) secondary echoes, dashed line is predicted from the measured primary echo using the parametric model, solid line the true measured secondary echo; (c) the residual (the difference between predicted and measured secondary echo).

\section{B. Accuracy of Modeling Wave Propagation}

When a good model for the frequency dependent bulk modulus is obtained, quantities describing the wave propagation such as phase velocity and attenuation can be computed.

In this section the phase velocity and attenuation are estimated using the bulk modulus model. The estimates obtained from the bulk modulus model are compared with estimates obtained from a non-parametric spectral model. The nonparametric model is based on computing the ratio between the discrete Fourier transformed second- and first echoes, to obtain the spectra from which the attenuation and phase velocity are estimated.

In Fig. 7 the mean value and the estimated standard deviation are shown for 50 attenuation estimates in oxygen, ethane and a mixture of $80 \%$ oxygen $20 \%$ ethane. Comparing the attenuation estimates, obtained from both models, a good agrement can be observed in the high SNR region. However, outside this region their similarities end, mostly due to the erratic behavior of the non-parametric method at low SNR, see [7]. This behavior can also be seen in the estimated standard deviation plots, as a decrease in accuracy compared to the bulk modulus model.

The plots are shown in the frequency region $[0.8,1.2] \mathrm{MHz}$

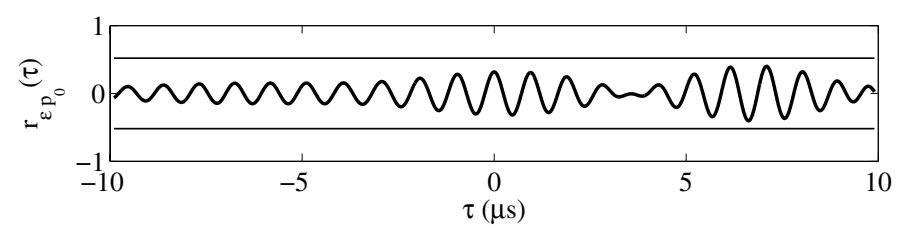

Fig. 6. Analysis of the residual in Fig. (5). Independence (correlation) test of the cross-correlation function between the residual $\varepsilon(n)$ and the first echo $p(0, n)$. The horizontal lines mark the $99 \%$ confidence region.
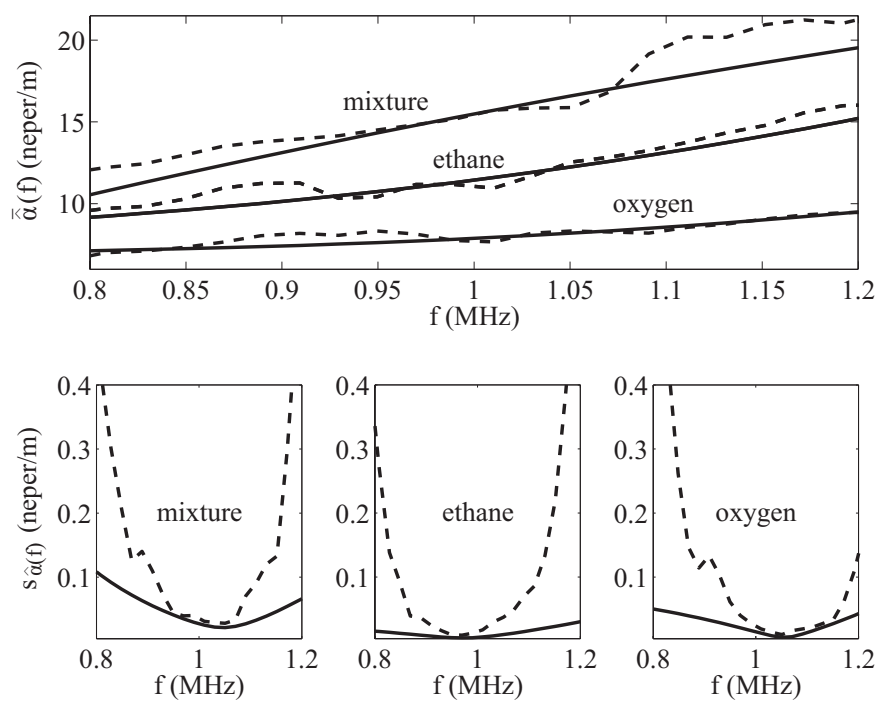

Fig. 7. Mean value $\overline{\hat{\alpha}}(f)$ and estimated standard deviation $s_{\hat{\alpha}(f)}$ of 50 attenuation estimates $\hat{\alpha}(f)$, where $\hat{\imath}$ denotes estimate. The attenuation $\alpha(f)$ is estimated with the bulk modulus model (solid lines) and the non-parametric model (dashed lines).

which corresponds to the $40 \mathrm{~dB}$ bounded spectrum bandwidth [8] of the first echo.

The phase velocity estimates are close to non-dispersive in the frequency region $[0.8,1.2] \mathrm{MHz}$ and the results are presented for $f_{0}=1 \mathrm{MHz}$ (transducer center frequency) in Table I. In Table I the phase velocity estimates are compared using: the parametric bulk modulus model $(P)$; the non-parametric spectral method $(N P)$; and standard crosscorrelation $(X)$. The mean values and estimated standard deviations coincides using the parametric model and crosscorrelation. For the non-parametric model, a large mean value offset can be seen together with a 100 times higher standard deviation. This high inaccuracy is mainly produced in the phase unwrapping step, when estimating the phase velocity using a non-parametric technique.

\section{Discussion}

The assumption that the residual is uncorrelated with the first echo is only valid for situations where the unknown input disturbance is negligible (here input and output refers to the first- and second echo). In presence of input disturbances or un-modeled non-linearities, which is often the case in reality, a natural correlation appears even though a good model is used. 
TABLE I

MEAN VALUE $\overline{\hat{c}}_{p}(f)$ AND ESTIMATED STANDARD DEVIATION $s_{\hat{c}_{p}(f)}$ OF 50 PHASE VELOCITY ESTIMATES $\hat{c}_{p}(f)$ (EVALUATED AT $\left.f_{0}=1 \mathrm{MHZ}\right)$. THE PHASE VELOCITY $c_{p}(f)$ IS ESTIMATED USING: THE PARAMETRIC MODEL (P); STANDARD CROSS-CORRELATION

(X); AND THE NON-PARAMETRIC MODEL (NP).

\begin{tabular}{|c|c|c|c|}
\hline method & $\begin{array}{c}\text { oxygen } \\
\overline{\hat{c}}_{p}\left(f_{0}\right) \pm s_{\hat{c}_{p}} 10^{3}\end{array}$ & $\begin{array}{c}\text { mixture } \\
\overline{\hat{c}}_{p}\left(f_{0}\right) \pm s_{\hat{c}_{p}} 10^{3}\end{array}$ & $\begin{array}{c}\text { ethane } \\
\overline{\hat{c}}_{p}\left(f_{0}\right) \pm s_{\hat{c}_{p}} 10^{3}\end{array}$ \\
\hline$T$ & $\overline{323.4 \pm 3.3}$ & $=3.222$ & $\overline{29}$ \\
\hline$X$ & 32 & 324 & 42 \\
\hline$N P$ : & $325.3 \pm 679.3$ & $326.1 \pm 759.2$ & $298.0 \pm 463.6$ \\
\hline
\end{tabular}

Considering the criterion of fit, improvements can be achieved by weighting it with the inverse covariance matrix of the noise or using an errors in variables approach, see [9]. Since we are dealing with repeated experiments with the same excitation signal, it is possible to get an estimate of the covariance matrix for the experimental noise.

In the minimization process, faster iteration can be achieved by deriving an analytical expression for the gradient (and Hessian) of Eq. 9.

\section{CONCLUSIONS}

In this paper we have showed how the combined effect of the absorption mechanisms present in a fluid can be described by modeling the bulk modulus as a series of mechanical submodels. The model's ability to describe pulse-echo measurements in oxygen, ethane and mixtures of the two, is validated using residual analysis and cross-validation. Compared to nonparametric methods the proposed structure gives, not only higher accuracy when modeling wave propagation in complex fluids, but also contributes to the understanding of the underlying dissipation mechanisms present in fluids.

\section{ACKNOWLEDGEMENTS}

This research was performed under grants from The Swedish Energy Agency and the The Kempe Foundation. The authors would also like to express their sincerest gratitude towards Dr. Pär-Erik Martinsson for valuable discussions and the experimental data.

\section{REFERENCES}

[1] L. E. Kinsler, A. R. Frey, A. B. Coppens, and J. V. Sanders, Fundamentals of Acoustics. New York: Wiley, 3rd ed., 1982.

[2] J. Shao, "Linear model order selection by cross-validation," J. Am. Stat. Assoc., vol. 88, pp. 486-494, June 1993.

[3] L. Ljung, System Identification: Theory for the User. Englewood Cliffs: Prentice-Hall, 2nd ed., 1999.

[4] G. Casula and J. M. Carcione, "Generalized mechanical model analogies of linear visoelastic behaivour," Bollettino Di Geofisica Teoretica Ed Applicata, vol. 34, no. 136, pp. 235-256, 1992.

[5] W. Murray, ed., Numerical Methods for Unconstrained Optmization. New York: Academic Press, 1972.

[6] G. S. Kino, Acoustic Waves: Devices, Imaging, and Analog Signal Processing. Englewood Cliffs: Prentice-Hall, 1987.

[7] L. Ljung, "On the estimation of transfer functions," Automatica, vol. 21, no. 6, pp. 677-696, 1985.

[8] L. W. Couch, Digital and Analog Communication Systems. Englewood Cliffs: Prentice-Hall, 6th ed., 2001.
[9] R. Pintelton and J. Schoukens, System Identification: A Frequency Domain Approach. IEEE Press, 2001. 